\title{
Predictors of Quality of Life in Infertile Couples
}

\author{
Zahra Royani ${ }^{1}$, Mohammad Heidari ${ }^{2}$, Mahboubeh Vatanparast ${ }^{3}$, Farideh Yaghmaei $^{4}$, \\ Athareh Kalantari Sarcheshme ${ }^{5}$, Jamileh Khademi Majomerd ${ }^{6}$ \\ ${ }^{1}$ Department of Nursing Education, Paramedical School, Golestan University of Medical Sciences, Gorgan, Iran, ${ }^{2}$ Community-Oriented \\ Nursing Midwifery Research Center, Shahrekord University of Medical Sciences, Shahrekord, Iran, ${ }^{3}$ Department of Reproduction Biology, \\ Rafsanjan University of Medical Sciences, Rafsanjan, Iran, ${ }^{4}$ Department of Nursing, Zanjan Branch, Islamic Azad University, Zanjan, Iran, \\ ${ }^{5}$ Department of Critical Care Nursing, School of Nursing Midwifery, Yazd Shahid Sadoughi University of Medical Sciences, Yazd, Iran, \\ ${ }^{6}$ Department of Midwifery, Reproductive Sciences Institute, Shahid Sadoughi Medical Sciences University, Yazd, Iran
}

Objectives: This study was conducted with the aim of examines the quality of life of infertile couples and their relationship with the practical resilience of infertile couples referring to Yazd's centers of infertility.

Methods: This research is a descriptive-correlational study. The research population consisted of all infertile couples who referred to Infertility Centers in Yazd, Iran in the winter of 2016. Sampling was conducted in a non-random and accessible manner. The instrument used in the research included a) demographic information questionnaire, b) Conner and Davidson's Resilience Scale, and c) quality of life infertile couples questionnaire. Data were analyzed by SPSS software version 17 at a significant level of $P<$ 0.05. To describe the data, descriptive statistics methods were used and the inferential statistics (Pearson correlation coefficient, regression, independent $t$ test, and variance analysis) were used to test the research hypotheses.

Results: People (202 couples) participated in this research. Three variables of resilience $(\beta=0.04, P=0.04)$, gender $(\beta=-0.22, P<$ $0.001)$, and education level $(\beta=0.21, P<0.001)$ had a prediction coefficient and there was a significant relationship with quality of life.

Conclusions: This study showed that resilience, gender, and education predict the quality of life of infertile couples. In the infertile couples counseling program, resilience should be considered as a coping factor. (J Menopausal Med 2019;25:35-40)

Key Words: Infertility · Quality of life · Couples

\section{Introduction}

Having a child is one of the life beauties. Individuals wishing to have children not only enjoy but also have a valuable memory of themselves. ${ }^{1}$ Infertility is regarded as a growing problem in all cultures and societies, and almost all over the world, and affects about $10 \%$ to $15 \%$ of couples in a reproductive age. This problem is likely to increase significantly in the near future as a result of the increasing num- ber of women delaying childbirth. ${ }^{2}$ According to the previous studies, nearly 50 million to 80 million people worldwide experience infertility. ${ }^{3}$ In addition, since most of infertility treatments focus on medical treatment and social and emotional aspects such as mental disorders and stresses related to infertile people have not been reported, the related studies have dramatically increased on the psychological dimensions of infertility during recent decades. Psychological problems reduce the patient's physical performance and response to

Received: August 29, 2018 Revised: September 18, 2018 Accepted: October 13, 2018

Address for Correspondence: Mohammad Heidari, Community-Oriented Nursing Midwifery Research Center, Shahrekord University of Medical Sciences, Ayatollah Kashani Blvd, Shahrekord 8815713471, Iran

Tel: +98-91-3185-0128, Fax: +98-38-1333-5652, E-mail: Heidari@skums.ac.ir

ORCID: https://orcid.org/0000-0001-7767-5645 
infertility treatment. ${ }^{4}$ These factors reduce the likelihood of a stable pregnancy with changes in cortisol and heart rhythm. ${ }^{5}$ In general, fertility status and its related factors have been effective on the quality of life by creating social psychological stress, reducing life satisfaction, increasing marital problems and reducing sexual self-confidence, as well as reducing sexual and marital satisfaction, which lead to a change in the quality of life. In addition, it is regarded as one of the most important issues in the world and has been presented as a measurable scale of treatment outcomes over the last few decades ${ }^{6}$ so that this assessment can be part of the treatment evaluation. The fertility status and its related factors lead to a change in the quality of life by inducing mental stress. ${ }^{6,7}$ In fact, the quality of life is a feeling of goodness and results from the satisfaction or dissatisfaction of various aspects of life which are important to the person. ${ }^{8,9}$ It includes health, occupational, socio-economic, psychosocial, and familial areas, and it is important as a scale of health care measurement. ${ }^{6,10,11}$ It is believed that this factor is one of the factors which may affect the outcome of infertility treatment. ${ }^{4}$

Despite the heavy emotional burden of infertility diagnosis, it does not mean that all couples are emotionally disappointed and only $20 \%$ to $25 \%$ of couples use counseling services if presented. The rest of the population seems to cope with resources to save the infertility crisis although the exact nature of these resources is not well-known. However, "resilience" can be an appropriate concept in this context. Resilience is defined as the mental ability of individuals to resist and adapt to life-threatening events. ${ }^{12}$ In fact, a person with resilience is remedial and flexible, adapts to the changes in his environment, and returns to recovery as the stressors quickly removed, and vice versa, those with lower resilience can adapt themselves less to new situations. ${ }^{13}$ In infertile couples, this factor is a protective factor against psychological distress and improving quality of life. ${ }^{14}$ In other words, couples who have a higher quality of life despite infertility are psychologically tolerant of relatively high stress. ${ }^{15}$ The feelings of people about the quality of life depend on the situation and time and change by the way they live. Considering that the factors related to quality of life vary in different societies and cultures, fertility is of great importance in socio-cultural terms and the studies on the impact of infertility on the quality of life had contradicted results in Western countries. ${ }^{5}$ Quantitative studies are available in eastern countries such as Iran. In addition, the correlation between resilience and quality of life in infertile couples has not been investigated yet. Therefore, the present study aimed to determine the correlation between resilience and quality of life in infertile women referring to centers of infertility in Yazd, Iran, 2015.

\section{Materials and Methods}

\section{Study design}

From March 2015 to June 2015, we asked from couples who referred to infertility center in Yazd to participate in this cross-sectional study. Participants were selected through convenience sampling. By the end June, 202 couples completed a questionnaire while waiting for their medical treatments. The criteria for including the study included: having satisfaction to participate in the research and failure to become pregnant at least one year after the decision to become pregnant. The criteria for excluding the study included having a history of admission to the psychiatric ward.

\section{Ethics statement}

The present study was approved by Ethical Committee of Medical Sciences University of Yazd (ethics code: 13.94.23). Also, after selecting the eligible participant, the researcher was introduced to them and the objectives of the study were elaborated for the participants. The informed consent was obtained from the subjects and they were assured that their information will remain confidential.

\section{Data collection}

The data collection was conducted with an introduction letter from the research deputy and with permission and coordination with the authorities in Infertility Center. The instruments used in the study are as follows:

A. The demographic information questionnaire includes age, sex, education, and duration of infertility.

B. Conner and Davidson's Resilience Scale (CDRS) which includes 25 Likert-scale options (never, rarely, sometimes, often, and always). Table 1 indicates the scores 
Table 1. Mean quality of life based on demographic variables

\begin{tabular}{|c|c|c|}
\hline Variable & Quality of life & $P$ value \\
\hline \multicolumn{3}{|l|}{ Age (y) } \\
\hline$<25$ & $179.98 \pm 24$ & 0.3 \\
\hline $25-30$ & $185.25 \pm 23.5$ & \\
\hline $31-35$ & $186.83 \pm 22.7$ & \\
\hline$>35$ & $183.21 \pm 23.59$ & \\
\hline \multicolumn{3}{|l|}{ Sex } \\
\hline Female & $169.54 \pm 16.92$ & $<0.01$ \\
\hline Male & $200.2 \pm 18.44$ & \\
\hline \multicolumn{3}{|l|}{ Level of education } \\
\hline Illiterate or elementary & $173.97 \pm 16.5$ & $<0.001$ \\
\hline Less than college & $183.5 \pm 24.6$ & \\
\hline College or higher & $189.41 \pm 22.8$ & \\
\hline \multicolumn{3}{|l|}{ Duration of infertility (y) } \\
\hline$<5$ & $185.2 \pm 23.5$ & $<0.07$ \\
\hline $5-10$ & $185.22 \pm 23.4$ & \\
\hline$>10$ & $183 \pm 23$ & \\
\hline
\end{tabular}

The data is presented as mean \pm standard deviation.

for each option. In order to get the total score of the questionnaire, the total score of all the questions is summed up. This score will range from 0 to 100. The higher the score, the greater the resilience of the respondent will be, and vice versa. The cut-off point for this questionnaire is a score of 50. In other words, the score above 50 is for people with resilience and when the score is above 50, the resilience of the person is higher too and vice versa. This scale was standardized in Iran by Mohammadi et al. ${ }^{16}$ Cronbach's alpha was used to determine the reliability of the CDRS and a reliability coefficient was obtained 0.89 (9 and 13).

C. Quality of life questionnaire for infertile couples was designed by Yaghmaei et al. ${ }^{17}$ This questionnaire has 72 questions including physical, psychological, spiritual and religious, economic, emotional, sexual and social factors with 5-point Likert answers from I totally agree to totally disagree. The face validity of the questionnaire was assessed by a survey of 20 experts and infertile couples. In order to measure reliability, internal consistency (Cronbach's alpha) and re-test were used. Finally, the Cronbach's alpha and the reliability coefficient was 0.81 was 0.89 , respectively. ${ }^{17}$

\section{Data analysis}

Data were analyzed by SPSS software version 17 (SPSS Inc., Chicago, IL, USA) at a significant level of $P<0.05$. Using the Kolmogorov-Smirnov test for data normalization and to describe the data, descriptive statistics methods were used and the inferential statistics (Pearson correlation coefficient, regression, independent $t$ test, and variance analysis) were used to test the research hypotheses.

\section{Results}

Four hundreds and four people participated in this study (202 couples), among whom 38.1\% were in the age group of 25 to 30 years, $42.6 \%$ had university education, $43.6 \%$ had a period of 5 to 10 years of infertility. Participants of $34.4 \%$ were as a result of male infertility, and $64.4 \%$ reported a modest economic outlook. Participants of $65.5 \%$ had the ability to pay for infertility treatment (Table 1).

The means of total quality of life, physical dimension, psychological dimension, religious dimension, economic dimension, sexual satisfaction, emotional dimension, and social dimension were $184 \pm 23.36,26.43 \pm 6$, $70.8 \pm 13.1$, $18.85 \pm 3.7,14.9 \pm 3.31,15.15 \pm 4.1,15.68 \pm 4.9$, and $23.3 \pm 6.6$, respectively. Based on the results, a significant difference was observed in the relationship between demographic variables and quality of life, mean of gender variables $(P<0.01)$ and education $(P<0.001)$. Thus, the average quality of life in men was $202.2 \pm 18.44$ and those with university education were higher (189.41 \pm 22.8$)$.

The mean total score of resilience was $63.65 \pm 15.51$. In studying the correlation between the quality of life and resilience, a significant relationship was observed $(P=0.008$, $r=0.13)$. Regarding the factors related to the quality of life, linear regression method was applied. The three variables of resilience $(\beta=0.04, P=0.04)$, gender $(\beta=-0.22, P<0.001)$, and educational level $(\beta=0.21, P<0.001)$ had a significant relationship with the quality of life (Table 2). 
Table 2. Estimation of regression coefficients of factors related to quality of life

\begin{tabular}{lcccc}
\hline \multicolumn{1}{c}{ Variable } & $\beta$ & Standard error & $\mathrm{t}$ & $P$ value \\
\hline Flexibility of action & 0.9 & 0.071 & 2.040 & 0.042 \\
Gender & -0.22 & 1.870 & -4.827 & 0.00 \\
Level of education & 0.21 & 1.550 & 4.522 & 0.00 \\
\hline
\end{tabular}

\section{Discussion}

In this study, $34.6 \%, 63.3 \%$, and $2.1 \%$ had a neutral quality of life, positive quality of life, and a completely positive quality of life. The results are consistent with the study results of Aliyeh and Laya. ${ }^{18}$ In addition, $38.1 \%$ of the subjects had moderate quality of life and nearly half of the people with good and excellent quality of life. The present study showed that there is a direct relationship between the resilience and quality of life of infertile couples, which increases the quality of life in these individuals by increasing the flexibility of the activity. The results of this study are in line with the results of other studies such as Herrmann et al. ${ }^{15}$ They performed a study in Germany in 2011 called functionality flexibility as a protective agent against the particular stress of infertility and the quality of life and found that high level of resilience in couples was accompanied with all aspects of quality of life and low scores in all the subset of the "List of Fertility Problems" was accompanied by the exception of the need for parents in women, and only with the concern of surrounding people in men. In general, they stated that resilience is a protective factor against the particular stress of infertility and the quality of life. ${ }^{15}$ Also, Barani Ganth et al. ${ }^{19}$ reported that resilience is one of the predictors for marital satisfaction. They emphasized that resilience can help couples deliberately solve problems and create a larger picture of mutual respect and solidarity. In the context of marriage, it is very likely that couples enter deep debates, and if there is no resilience, the disagreements and feelings of harm will lead to a cold relationship. Resilience resulted in creating these impasse and cold situations in marital relationships.

Further, the findings indicated that the effects of infertility on the quality of life in women are higher than that of men. In fact, it was found that infertile women have a lower quality of life. According to Aslzaker et al. ${ }^{4}$ and Andrews et al., ${ }^{20}$ high levels of stress in infertile women are related to reducing marital function and loss of quality of life, irrespective of the its reasons. Furthermore, the findings are congruent with other study results. ${ }^{2,21,22}$ Regarding the justification for these findings the cultural factors of each society may be involved, for example, in Korean and Japanese culture. The infertile woman means the "woman made of rock", which is regarded as "a disadvantaged woman" in the South Indian language. In other words, she is not allowed to attend the meetings, weddings and birthdays of the child. Most Indian women are obsessed with childbirth several months after marriage. In contrast to men who are infertile, they manage this situation better. From their point of view, infertility is a social stigma which only affects women. ${ }^{16}$ In Iranian culture, childbearing is also very important for women. On the other hand, low quality of life in women can be attributed to the importance of having a child for women and more involvement in infertility and its treatment.

Regarding other findings in the present study, the existence of higher quality of life was higher in people with higher education so that those with university education had higher quality of life than those with lower education, maybe due to the fact that they were less embarrassed than those with lower education. ${ }^{2,23}$ On the other hand, people with a higher education level use problem-solving skills better. After passing years of education, it seems that they learn how to deal with daily stressors and use new methods to deal with the problem. Finally, no significant relationship was observed between the quality of life, the cause of infertility, and the duration of infertility. In this regard, Aslzaker et al. $^{4}$ achieved similar results. However, in the study of Rashidi et al., ${ }^{2}$ the quality of life was reported as higher in male infertility. It seems that infertility is a similar experience which is understood in both women and men. 
The results of the study indicated that resilience can predict the quality of life among infertile couples against infertility stress which can be confirmed by conducting further studies on the quality of life and resilience before, during and after the treatment of infertility. In addition, infertile women have lower quality of life than that of infertile men. In order to improve the quality of life among these individuals, and increase the mental health and health of infertile patients, especially infertile women, psychological counseling, especially psychological supportive therapy, can be effective in reducing the psychological problems of this group of patients and improving their quality of life by emphasizing the skills used for resilience.

One of our study limitations was that we didn't have these data in the fertile couple, to compare the infertile results with a norm population in the society. It is proposed that this work will be done, simultaneously, in the fertile and infertile group.

\section{Conflict of Interest}

No potential conflict of interest relevant to this article was reported.

\section{References}

1. Ehsanpour S, Mohsenzadeh N, Kazemi A, Yazdani M. The relation between social support and stress in treatment of infertility in infertile couples referred to infertility centers of Isfahan in 2007. Iran J Nurs Midwifery Res 2009; 14: 51-5.

2. Rashidi B, Montazeri A, Abedineia N, Shariat M, Afshari M, Ramezanzadeh F. Heath related quality of life in Iranian couples receiving IVF/ICSI treatment. Payesh 2012; 11: 3859.

3. Zamani N, Ghasemi M, Jokar E, Khazri Moghadam N. Comparison of depression and life quality of fertile and infertile women and those with frequent abortions. J Babol Univ Med Sci 2013; 15: 78-83.

4. Aslzaker M, Pourshahbaz A, Bagheri Lankarani N, Mohammadkhani P, Geranmayepour S. Effects of infertility stress, psychological symptoms, and quality of life on predicting success rate of IVF/ICSI treatment in infertile women. Practice Clin Psychol 2016; 4: 275-81.
5. Globevnik Velikonja V, Lozej T, Leban G, Verdenik I, Vrtačnik Bokal E. The quality of life in pregnant women conceiving through in vitro fertilization. Slovenian J Public Health 2016; 55: 1-10.

6. Ridenour AF, Yorgason JB, Peterson B. The infertility resilience model: assessing individual, couple, and external predictive factors. Contemp Fam Ther 2009; 31: 34-51.

7. Bahadori M, Ghardashi F, Izadi AR, Ravangard R, Mirhashemi S, Hosseini SM. Pre-hospital emergency in Iran: a systematic review. Trauma Mon 2016; 21: e31382.

8. Heidari M, Shahbazi S, Ghodusi M. Evaluation of body esteem and mental health in patients with breast cancer after mastectomy. J Midlife Health 2015; 6: 173-7.

9. Heidari M, Shahbazi S, Ghafourifard M, Ali Sheikhi R. Prediction of marital satisfaction based on emotional intelligence in postmenopausal women. J Menopausal Med 2017; 23: 196-201.

10. Shirvani M, Heidari M. Quality of life in postmenopausal female members and non-members of the elderly support association. J Menopausal Med 2016; 22: 154-60.

11. Abolghasem-Gorji H, Bathaei SA, Shakeri K, Heidari M, Asayesh $\mathrm{H}$. The effect of religiosity on quality of life in Muslim patients with heart failure: a study in Qom, the religious capital of Iran. Ment Health Relig Cult 2017; 20: 21728.

12. Connor KM, Davidson JR. Development of a new resilience scale: the Connor-Davidson Resilience Scale (CD-RISC). Depress Anxiety 2003; 18: 76-82.

13. Harter JK, Schmidt FL, Keyes CL. Well-being in the workplace and its relationship to business outcomes: a review of the Gallup studies. In: Keyes CLM, Haidt J, editors. Flourishing: positive psychology and the life well-lived. Washington, DC: American Psychological Association; 2003. pp. 20524.

14. Silber S, Geisler JH, Bolortsetseg M. Unexpected resilience of species with temperature-dependent sex determination at the Cretaceous-Palaeogene boundary. Biol Lett 2011; 7: 2958.

15. Herrmann D, Scherg H, Verres R, von Hagens C, Strowitzki T, Wischmann T. Resilience in infertile couples acts as a protective factor against infertility-specific distress and impaired quality of life. J Assist Reprod Genet 2011; 28: 11117.

16. Mohammadi M, Jazayeri A, Rafiei A, Jokar B, Pourshahnaz A. A study on the factors affecting resilience in people at risk of substance abuse. Modern Psych Res 2005; 1: 203-24.

17. Yaghmaei F, Mohammadi S, Alavimajd H. Developing "quality of life in infertile couples questionnaire" and measuring its psychometric properties. J Reprod Infertil 2009; 
10: $137-43$.

18. Aliyeh G, Laya F. Quality of life and its correlates among a group of infertile Iranian women. Med Sci Monit 2007; 13: CR313-7.

19. Barani Ganth D, Thiyagarajan S, Nigesh. Role of infertility, emotional intelligence and resilience on marital satisfaction among Indian couples. Int J Appl Psych 2013; 3: 31-7.

20. Andrews FM, Abbey A, Halman LJ. Is fertility-problem stress different? The dynamics of stress in fertile and infertile couples. Fertil Steril 1992; 57: 1247-53.

21. Goker A, Yanikkerem E, Birge O, Kuscu NK. Quality of life in Turkish infertile couples and related factors. Hum Fertil (Camb) 2018; 21: 195-203.

22. Xiaoli S, Mei L, Junjun B, Shu D, Zhaolian W, Jin W, et al. Assessing the quality of life of infertile Chinese women: a cross-sectional study. Taiwan J Obstet Gynecol 2016; 55: 244-50.

23. Donkor ES, Sandall J. The impact of perceived stigma and mediating social factors on infertility-related stress among women seeking infertility treatment in Southern Ghana. Soc Sci Med 2007; 65: 1683-94. 